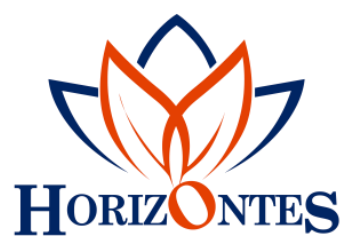

www.revistahorizontes.org

\title{
Una mirada gerontológica hacia la política social y educación permanente en latinoamérica
}

A gerontological look at social policy and permanent education in latin america

Um olhar gerontológico sobre a política social e a educação permanente na américa latina

ARTICULO DE INVESTIGACIÓN

Arantxa Ortiz-Hernández

psicarantxaortiz@gmail.com

ORCID: 0000-0003-1006-7009
Melina Rodríguez-Díaz

melina_rd@yahoo.com.mx

ORCID: 0000-0001-6734-7664

Maestría en Gerontología, Departamento de Salud Pública, Centro Universitario de Ciencias de la Salud, Universidad de Guadalajara, Guadalajara, México

Recibido 04 de febrero 2021 | Arbitrado y aceptado 16 de febrero 2021 | Publicado en marzo 2021

\section{RESUMEN}

La educación permanente es una propuesta innovadora que aspira la inclusión y optimización de los recursos internos de las personas en su aprendizaje. Su presencia en las convenciones internacionales como un derecho señala la adopción de ella para la elaboración de políticas sociales enfocadas a la población mayor de México y en Latinoamérica. El objetivo de este estudio fue la búsqueda y comparación de los parámetros de los programas enfocados a la educación permanente. La búsqueda de información fue por medio de la revisión diferentes documentos oficiales de instituciones públicas de México y y otros países latinoamericanos. A pesar de que actualmente existen planes y programas que acatan este requerimiento, se desconoce su estado de implementación y los resultados que verdaderamente tiene en la población de adultos. Es necesaria la participación colectiva de instituciones públicas y privadas para un desarrollo educativo de calidad en las personas adultas mayores.

Palabras clave: Educación permanente; personas adultas mayores; política social; gerontología; educación a adultos

\section{ABSTRACT}

Lifelong education is an innovative proposal that aspires to the inclusion and optimization of people's internal resources in their learning. Its presence in international conventions as a right signal the adoption of it in the making of social policies focused on the elderly population of Mexico and Latin America. The aim of this study was the search and comparison of the parameters of the programs focused on permanent education. The search for information was by reviewing different official documents from public institutions in Mexico and other Latin American countries. Even though there are currently plans and programs that follow this requirement, is currently unknown the implementation status and the results it truly has in the adult population. The collective participation of public and private institutions is necessary for quality educational development in older adults.

Key words: Lifelong education; older adults; education for adults; social policies; gerontology 


\section{RESUMO}

A educação permanente é uma proposta inovadora que visa incluir e otimizar os recursos internos das pessoas em sua aprendizagem. Sua presença em convenções internacionais como um direito indica sua adoção para a elaboração de políticas sociais voltadas para a população idosa do México e da América Latina. 0 objetivo deste estudo foi a busca e comparação dos parâmetros dos programas voltados para a educação permanente. A busca de informações se deu por meio da revisão de diferentes documentos oficiais de instituições públicas do México e de outros países da América Latina. Apesar de já existirem planos e programas que atendam a essa exigência, não se sabe qual é o seu estágio de implantação e quais são os resultados que realmente têm na população adulta. A participação coletiva de instituições públicas e privadas é necessária para o desenvolvimento educacional de qualidade dos idosos.

Palavras-chave: Educação ao longo da vida, idosos, política social, gerontologia, educação de adultos

\section{INTRODUCCIÓN}

El proceso de envejecimiento demográfico, presente en Latinoamérica, adquiere gran interés debido a las transformaciones sanitarias, económicas y sociales que se desarrollarán para satisfacer las necesidades de la población. Específicamente en México, en el año 2018 residían 15.4 millones de personas adultas mayores en el país, lo cual representaba el $12.3 \%$ de la población total (Instituto de Estadística y Geografía, 2019), de acuerdo con las aproximaciones del Consejo Nacional de Población (2012) para el 2050 esta cifra aumentara un $21.5 \%$.

Para enfrentar las diferentes directrices que se presentan ante la situación de envejecimiento, en el cual Osorio (2016) designa la manifestación de múltiples problemas de desigualdad, pobreza y falta de servicios sociales; los países necesitan desarrollar entornos asistenciales favorables para mejorar la calidad de vida de las personas adultas mayores (Organización Mundial de la Salud, 2010). La política social enfocada en la vejez responde a las necesidades de bienestar por medio de la implementación de medidas de solución ante cada ámbito específico (Roque, Azcuy y Toledo, 2015). En el caso de México, se ha transformado la manera en que el Estado concibe a la vejez, a la cual en recientes décadas se le ha atribuido la calidad de persona sujeta a derechos; de la cual se tiene un enfoque hacia asegurar la integridad, dignidad y fortalecimiento de la autonomía de los adultos mayores en un ámbito de igualdad (Instituto Nacional de las Personas Mayores, 2010). Con ello, Razo-González (2014) señala la promoción de acciones prospectivas que abarquen las necesidades especiales de este grupo en la cual las instituciones gubernamentales y los demás actores involucrados participen en la búsqueda de una verdadera integración social.

El acceso a la educación se le considera un factor importante para el alcance a una vejez más satisfactoria (Serdio, 2015), al considerarse como una actividad liberadora que impulsa a la persona a alcanzar su desarrolla personal y comunitario, de igual manera se le ha relacionado como un indicador de prevención ante declives en su actividad cerebral (Bermejo, 2010); sin embargo su abordaje es complicado debido a la heterogeneidad grupal proveniente de las experiencias de vida de cada persona mayor; por ende se requiere de propuestas que se distancien del modelo educativo tradicional para así otorgar espacios inclusivos de 
aprendizaje y el abordaje de temas integrales para la vejez (García, 2007).

Las características necesarias para un modelo de formación en mayores, Mogollón (2012) refiere que se requiere un enfoque dirigido al adulto mayor, en la cual se incluyan actividades que le sean de interés a él y responda ante las necesidades de esta etapa de vida; una mentalidad social libre de prejuicios y enfocada a la participación; la oportunidad de satisfacer oportunidades que en años anteriores no lograron concretar y la promoción de una interacción intergeneracional. De esta manera el adulto mayor se convierte en un miembro contributivo y receptivo del entramado social en el que vive y se desarrolla.

\section{Planteamiento del problema}

Ante la necesidad de un marco de educación que promueva el desarrollo individual y social en el individuo, se origina la Educación Permanente [EP] que Valcárcel (2003) define como un proceso continuo que tiene el propósito de brindar a la persona la oportunidad de mantenerse actualizada respecto a las transformaciones poblacionales, económicas, políticas, tecnológicas, científicas, artísticas, socioculturales y ambientales en su contexto; de esta manera utiliza todo tipo de experiencias y actividades para lograr el máximo desarrollo individual y social que le sea posible. Los principios de la EP consisten en la coordinación e innovación de los recursos que el individuo tiene disponibles con los cuales podrá formarse a lo largo de su ciclo vital; desde un marco individual hasta en el desarrollo de la comunidad donde se desenvuelve; cabe destacar que se rige desde un marco inclusivo en el cual se busca prevenir y abordar necesidades de determinadas edades y grupos sociales (Medina, Llorent y Llorent, 2013). En sí, Sabán (2010) la define como un modelo de educación que intenta abordarla desde todos los niveles de vida para que sea recibida y ejercida por cada persona.

La EP, a nivel internacional, es una iniciativa atractiva por su énfasis en el desarrollo individual y social, la apertura a contextos no formales y la utilización del conocimiento práctico como recurso disponible para la persona (Reyes y Machado, 2017). Ante una creciente demanda de la población adulta mayor para programas educativos que aborden temas más allá de la educación tradicional basada solamente en la alfabetización; Valle (2014) refiere que la EP promueve la inclusión de este grupo etario en el desarrollo cultural por medio de oportunidades de relación y comunicación social desde una perspectiva positiva, activa, participativa y de autorrealización. Gracias a los esfuerzos por parte de la UNESCO y la Comisión de Cultura y Educación del Consejo de Europa, se ha impulsado la idea de una educación inclusiva desde un parámetro universal (Tünnermann, 2010), por ende diferentes protocolos y convenciones han integrado a la EP dentro de los temas a abordar con relación a los derechos en la vejez:

- La Segunda Asamblea Mundial sobre el Envejecimiento (2002) señala la igualdad de oportunidades dentro de la EP, al ser una vía de acceso para actividades de capacitación y readiestramiento en zonas rurales.

- La Estrategia Regional de implementación para América Latina y el Caribe del Plan de Acción Internacional de Madrid sobre el 
Envejecimiento (2003) refiere como consideración general el acceso a la EP para disminuir brechas generacionales por medio de dar una igualdad de oportunidades para lograr un mayor desarrollo social.

- La Declaración de Brasilia (2007) alude a impulsar el acceso a la EP en cada etapa de vida en el ciudadano.

- La Carta de San José sobre los derechos de las personas mayores de América Latina y el Caribe (2012) de igual manera la indica como un derecho, en el cual se enfoca desde una perspectiva de aprendizaje intercultural por medio del intercambio de conocimientos, cultura y valores.

- La Convención Interamericana sobre la Protección de los Derechos Humanos de las Personas Mayores (2015) señala la importancia en promover su acceso a grupos vulnerables y la satisfacción de sus necesidades, preferencias, aptitudes, motivaciones e identidad cultural.

Como se puede observar, se insiste en la creación de estrategias que impulsen el desarrollo comunitario y satisfagan las necesidades sociales, biológicas y psicológicas del adulto mayor con un enfoque en su proceso de envejecimiento. Debido a ello, el objetivo de este estudio es analizar las políticas sociales de México y otros países latinoamericanos hacia la Política social y Educación Permanente en los adultos mayores.

\section{METODOLOGÍA}

La búsqueda de información se realizó de la siguiente manera, primero se revisaron diferentes documentos oficiales de instituciones públicas de México, principalmente del Instituto Nacional de
Personas Mayores y el Instituto Nacional de la Educación de los Adultos, a fin de encontrar temas relacionados a vejez y EP; a pesar de que el segundo término suele confundirse con la educación continua y educación para adultos, solamente se escogieron propuestas que fueran acordes a las definiciones teóricas de la EP. Las palabras clave utilizadas fueron educación permanente, educación y educación en adultos. Por ende, en base a los criterios mencionados, se encontraron 6 documentos que especificaban una ley referente a la obtención de educación y dos programas enfocados a este modelo educativo, "Educación para la salud" del INAPAM y el "Modelo Educación para la Vida y el Trabajo" del INEA, ambos programas comunitarios dentro del territorio mexicano. De ahí, se buscó si existían páginas que detallaran que existiera alguna página oficial que estuviera al alcance de la población, la cual solo la INEA tenía. Lo siguiente fue hacer un comparativo entre los programas mexicanos a otros programas en Latinoamérica que tuvieran un mismo enfoque basado en la EP, siendo 14 documentos en total por 7 países.

\section{Derechos enfocados en la educación para adultos mayores}

En la Ley de Derechos de Personas Mayores (2002), en sus últimas reformas en el 2018, se señala en el artículo 5to el derecho preferente hacia la obtención de educación, además en siguientes párrafos se menciona la necesidad de abordar temas de especial interés para el adulto mayor tanto en su vida cotidiana como en un enfoque de capacitación laboral.

Dentro de los ejes rectores de la política pública nacional descritos por el Instituto Nacional de las Personas Adultas Mayores [INAPAM] (2010), la educación, junto a 
aspectos referentes a la EP, se presenta principalmente en el eje referente a un envejecimiento activo y saludable. En el texto se refiere la necesidad de espacios educativos para empoderar a los adultos mayores en el conocimiento de sus derechos y desarrollen las herramientas necesarias para alcanzar un bienestar. De igual manera, aboga hacia un aprendizaje enfocado en mantener la independencia y la participación comunitaria. En sí, la educación se maneja como un concepto que permea en cada una de las esferas del envejecimiento: social, biológica y psicológica. Las recomendaciones expuestas reiteran:

- La difusión de oferta educativa exclusiva para personas adultas mayores.

- Incrementar las oportunidades de acceso a la educación o actividades de formación constante.

- Realización de jornadas informativas en temas relacionados al envejecimiento o de interés para las personas mayores y sus familiares. Cabe especificar que el contexto planteado sea de apertura al diálogo entre participantes.

\section{RESULTADOS Y DISCUSIÓN}

\section{Programas de EP}

El INAPAM (2010) ha implementado programas de educación para la salud con una perspectiva preventiva de ciclo de vida. En sus dos líneas, enfocada para las personas adultas mayores y otros grupos de edades, se abordan temas de desarrollo personal, igualdad, protección de derechos y plan de vida futura, entre otros. Sus objetivos planteados son la promoción del autocuidado, sensibilización ante el envejecimiento e impulsar la convivencia intergeneracional. Los talleres se imparten en dependencias del INAPAM.

Por otra parte, el Instituto Nacional de la Educación de los Adultos [INEA] (2013) oferta el programa educativo "Modelo Educación para la Vida y el Trabajo [MEVyT]" el cual ofrece ofertas alternativas de educación continua y el abordaje de temas personales y comunitarios relacionadas a temas de interés en la población de 15 años en adelante. Sus objetivos son el rescatar experiencias personales y colectivas para construir otros aprendizajes además de desarrollar habilidades, actitudes $\mathrm{y}$ valores en sus estudiantes. Las características del programa son la flexibilidad y apertura en la estructura de módulos de aprendizaje.

La aplicación de cada módulo de aprendizaje se presenta en cuatro pasos: la recuperación de conocimientos previos, análisis de nueva información, comparación y reflexión para una reconceptualización hacia el cambio. El razonamiento crítico y la aplicación de los saberes en la vida diaria son productos que se esperan estimular en el transcurso de los módulos (INEA, 2017).

En el marco de la inclusividad, existen tres modalidades para acatar necesidades específicas de ciertos sectores de la población: en línea, Indígena Bilingüe y Braille.

- MEVyT en línea es la modalidad electrónica que facilita la obtención de conocimientos fuera de aulas, por medio de cursos ofertados en una plataforma se explican los temas con medios audiovisuales y la oportunidad de participar en una comunidad virtual (INEA, 2014).

- MEVyT Indígena Bilingüe presenta los temas pero orientados en la alfabetización al español. Las características lingüísticas 
de las personas se toman en suma consideración para la planeación del programa de aprendizaje (INEA, 2013).

- MEVyT Braille es similar a la modalidad anterior, solo que esta acata a las personas con debilidad visual y enseña el sistema de escritura braille en sus sesiones (INEA, 2012).

\section{Programas de EP en Latinoamérica}

En el contexto de Latinoamérica los programas de EP se han elaborado para atender las exigencias de su población, aunque en las convenciones la educación se presenta una necesidad, solo algunos países lo han abordado con un planteamiento de participación comunitaria y empoderamiento característico de la EP. A continuación se muestra una tabla que ilustra la información descrita.

Tabla 1. Programas de educación permanente en Latinoamérica

\begin{tabular}{|c|c|c|}
\hline Países & $\begin{array}{c}\text { Nombre de } \\
\text { programas/proyectos }\end{array}$ & Características \\
\hline Costa Rica & $\begin{array}{l}\text { Instituto de Educación } \\
\text { Comunitaria (IPEC) }\end{array}$ & $\begin{array}{l}\text { Departamento de Educación de Personas Jóvenes y Adultas. } \\
\text { - Objetivos: obtener mayor cobertura, equidad, inclusión y } \\
\text { calidad en los procesos educativos } \\
\text { - Se compone de: I nivel (I y II Ciclos), II nivel (III Ciclo) y III } \\
\text { nivel (Educación Diversificada), cursos libres y carreras } \\
\text { técnicas }\end{array}$ \\
\hline Ecuador & $\begin{array}{c}\text { Educación } \\
\text { Extraordinaria para } \\
\text { Personas con } \\
\text { Escolaridad Inconclusa }\end{array}$ & $\begin{array}{l}\text { Ministerio de Educación, Ley Orgánica de Educación } \\
\text { Intercultural, el Plan Nacional del Buen Vivir y el Plan Decenal. } \\
\text { - } \quad \text { Objetivos: Brindar oportunidad de concluir los estudios en } \\
\text { jóvenes y adultos que son parte de los grupos vulnerables } \\
\text { y excluidos del sistema educativo y del modelo económico, } \\
\text { social y político. } \\
\text { Responden a la heterogeneidad de los estudiantes y a sus } \\
\text { diversos contextos, con currículos y propuestas } \\
\text { pedagógicas diversificadas y servicios educativos } \\
\text { adecuados a sus necesidades y particularidades. } \\
\text { - Desarrollen competencias para la vida y el trabajo. } \\
\text { Lograr aprendizajes significativos en los estudiantes. }\end{array}$ \\
\hline $\begin{array}{c}\text { El } \\
\text { Salvador }\end{array}$ & $\begin{array}{c}\text { Programa de } \\
\text { Alfabetización y } \\
\text { Educación Básica para } \\
\text { la población Joven y } \\
\text { Adulta }\end{array}$ & $\begin{array}{l}\text { Ministerio de Educación. } \\
\text { - Comprensión de la lectura, la expresión escrita y el uso del } \\
\text { cálculo matemático básico } \\
\text { - Participación voluntaria de comunidades, gobiernos } \\
\text { locales, empresas privadas, iglesias } \\
\text { Objetivos: Garantizar el derecho a la educación } \\
\text { permanente en la población joven y adulta. } \\
\text { - Estrategias de atención para promover actitudes } \\
\text { favorables hacia la búsqueda de información, reflexión, } \\
\text { cooperación, organización, autogestión y cogestión. }\end{array}$ \\
\hline
\end{tabular}




\begin{tabular}{cccc}
\hline Países & $\begin{array}{c}\text { Nombre de } \\
\text { programas/proyectos }\end{array}$ & \multicolumn{1}{c}{ Características } \\
\hline Guatemala & Programa Nacional de & Ministerio de Educación, Dirección General de Educación \\
& Educación Alternativa & $\begin{array}{l}\text { Extraescolar. } \\
\text { - }\end{array}$ & Objetivos: contribuir a la ampliación de cobertura \\
& & educativa del subsistema de educación extraescolar por \\
& medio de modalidades semi presenciales y distancia. \\
& & Ofrece certificación de competencias adquiridas de manera \\
& & formal o informal por los estudiantes. \\
& & Una de sus ofertas es la Educación para la vida y el trabajo, \\
& que incluye diplomados vinculados a la formación humana \\
& y emprendimiento.
\end{tabular}

Paraguay Educación Permanente Ministerio de Educación y Ciencias.

- Tienen diferentes programas pero no hay acceso a sus páginas web

- Comprende tres ciclos y es considerada como una formación supletoria.

- Se desarrolla con clases presenciales y a distancia, generalmente en el turno noche.

- Las unidades operativas funcionan en diferentes locales, tales como escuelas, colegios oficiales, municipalidades, cooperativas, capillas religiosas, clubes, policiales $\mathrm{y}$ militares, etc. Programa Aprender
Siempre

Venezuela Educación de Jóvenes, Adultos y Adultas
Dirección de Educación

- Educación no formal para personas jóvenes y adultas.

- Acceso a propuestas educativas durante todas las etapas de la vida en diferentes contextos ambientales y culturales, con un fuerte enclave territorial.

- Dos líneas de trabajo generales: actividades educativas en contextos de encierro y actividades educativas en espacios surgidos en comunidad.

Ministerio del Poder Popular para la Educación

- Sus áreas de aprendizaje son educación media, diversificada y profesional, capacitación en Artes y Oficios.

- Promocionar una educación integral y de calidad, la apertura de espacios de encuentro y participación entre la comunidad estudiantil y la comunidad educativa, que promociona la creatividad, la convivencia, la participación, la solidaridad, la reflexión, a la vez que aboga por la conservación del medio ambiente, la salud integral, y el uso de las tecnologías de la información.

- Es del año 2008 y actualmente no está disponible la página web del Ministerio para revisar alguna actualización. 
Los diferentes países han intentado incluir programas de educación permanente en sus políticas de educación por medio de diversos alcances. En general, lo plantean como una variante de la educación para adultos y en temas enfocados al aprendizaje de oficios y alfabetización, de igual manera incluyen postulados de la EP: inclusión, aprendizajes significativos y planes que abordan las individuales de los adultos.

\section{Discusión}

\section{Situación actual de la EP}

A pesar de que el programa MEVyT promete un desempeño amplio en alcance y favorable en respecto a resultados educativos en la población, en sí existen pocas evaluaciones del desempeño. De acuerdo con los resultados obtenidos, la Facultad Latinoamericana de Ciencias Sociales (2008) refiere que existe un vacío de información en respecto a datos cualitativos sobre la experiencia de aprendizaje en el adulto, además que hay una posible discrepancia de orientación entre objetivos previstos en una educación formal tradicional a los establecidos en la EP, siendo el caso que aunque se espera una mayor flexibilidad y orientación hacia el aprendizaje experiencial en realidad se mantiene dentro de los parámetros de un programa de educación continua común. Otro factor preocupante es que el personal del INEA carece del perfil necesario para implementarlo en su totalidad, por lo que se presenta una deficiencia al momento de aplicarlo dentro de la población, siendo el caso más prominente en zonas con difícil acceso.

Por otra parte, la INEA (2009) evaluó el impacto utilizando un grupo experimental y de control para hacer un comparativo entre poblaciones de estudio. Las variables para analizar fueron autopercepción del interés y satisfacción, beneficios percibidos, cambios, empoderamiento y autoestima. Ambos grupos se compusieron de 1402 en experimental y 701 en control, con 15 años en adelante. Cabe destacar que en el documento no se mencionan el número de sesiones que los estudiantes participaron antes de la evaluación.

Los resultados obtenidos fueron los siguientes:

- Las personas mencionaron que las 3 razones principales para inscribirse en el programa son: mejora de condiciones de vida, superación académica y el papel de la educación en un mayor bienestar.

- Se presenta una mayor autosuperación y autopercepción de las capacidades.

- Autopercepción en la mejora de habilidades de lectura, escritura y razonamiento matemático.

- Leve índice de mejora en aprendizaje de habilidades de higiene, manejo de ingresos y autocuidado.

- Mayor aprendizaje de habilidades para la resolución de conflictos, en la toma de decisiones e inteligencia emocional.

En general se obtuvieron resultados favorables en las áreas de aprendizaje en lectoescritura y matemático además de un mayor crecimiento personal en temas emocionales. Se puede explicar la falta de cambio en las otras áreas de intervención debido al poco tiempo en que las personas asistieron a los talleres, aunque como se mencionó no se puede saber con certeza por la falta de información. 
Por otra parte, el programa de Educación para la Salud presenta un informe trimestral que solamente menciona tener un avance del 89.9\% entre enero y marzo (INAPAM, 2013). Los puntos abordados en los talleres y una visión más detallada acerca del proceso de aprendizaje que los adultos mayores no son mencionados. A pesar un programa educativo que debería tener el mayor enfoque gerontológico no hay una certeza que esté cumpliendo con los cometidos planeados por el mismo instituto.

Ambos programas, el MEVyT y el de Educación para la Salud plantean diversos aspectos de la EP en la cual se aboga ante una oportunidad educativa para desarrollar capacidades en diferentes ámbitos y de esta manera mantener un vínculo social en su comunidad, cabe destacar que el MEVyT centra sus aspectos en base a primeramente alfabetizar a la población inscrita en él para luego dar una formación de hábitos de vida junto a aprendizaje social y emocional. El tema de inclusión también es abordado con la opción de una modalidad braille. Por otra parte, el de Educación para la Salud se enfoca en la psicoeducación, en la cual se espera el proveer herramientas, en base a la experiencia del usuario, para que puedan mejorar su estilo de vida y así llevar a cabo una mayor autorrealización. Aunque las políticas señalan la utilización de la EP para fomentar un envejecimiento activo, dentro del país no se hace una mención específica del plan de trabajo para cada grupo de edad, el adulto y en la vejez, por lo que las singularidades que pueden portar cada etapa no son consideradas.

Al hablar de un panorama más amplio acerca de la EP, en Latinoamérica se ha propuesto la implementación por medio de programas relacionados a sus institutos públicos educativos, en general todos ellos intentan incluir las características de este modelo pero no logran abordarlo en extenso, siendo el punto en común de ellos el crear un espacio de inclusividad para sus ciudadanos. Sin embargo, se tiene una visión aún enfocada a la educación tradicional, en la búsqueda de dar a la persona un grado académico y no potenciar los conocimientos ya obtenidos en su curso de vida. México en comparación con otros países presenta las mismas limitaciones en cuánto a la metodología planteada.

\section{CONCLUSIONES}

En este trabajo se revisó la presencia de la educación permanente en los planes de políticas sociales en México y su desenvolvimiento en Latinoamérica. Las convenciones internacionales se encargan de establecer los parámetros para la mejora de calidad de vida de la población, de los cuales los países pueden trazar sus planes de acción a diferentes niveles. En general buena parte de los países buscan la implementar una educación para adultos más incluyente y dirigida al empoderamiento de los grupos sociales, aunque sus alcances pueden variar de acuerdo con su seguimiento de estos. México tiene, al menos en planeación, programas que capturan la esencia de la educación permanente y de los cuales se podrían obtener resultados completamente favorables en la superación personal y colectiva de las personas mayores. Sin embargo, como se puede observar no hay evaluaciones rigurosas de ellos por lo que es imposible comentar si realmente están acatando las necesidades de las personas involucradas.

Se requiere un verdadero involucramiento de las instituciones gubernamentales y la utilización de conocimiento científico para desarrollar programas de calidad en cada una de sus etapas, de esta manera se podrá conocer 
las capacidades y aprendizajes significativos de las personas adultas mayores. La capacitación de los facilitadores y equipo de trabajo se convierte en una necesidad para que estos programas funcionen, ya que ellos serán los que interactúen con las personas y apoyen en la expresión de sus experiencias. A su vez, la misma EP señala la importancia de la sociedad para impulsar la transformación de las capacidades intrínsecas por lo que una metodología dialéctica y participativa en imprescindible para su desarrollo en cada uno de los grupos.

Otro punto para retomar es la ausente representación gerontológica, en donde al adulto mayor se aglomera en el concepto "adulto" sin atender a las necesidades y características que puede tener cada etapa de vida, desde el joven hasta la ancianidad. Cada nueva generación de personas mayores en México tiene una mayor presencia política, económica, social por lo que las exigencias de una mejor atención en todos los niveles será una realidad al pasar los años, por ende es obligatorio la búsqueda de calidad en la aplicación de las políticas educativas contemporáneas.

\section{REFERENCIAS}

Bermejo, L. (2010b). Pedagogía gerontológica y buenas prácticas socioeducativas con personas mayores. En L. Bermejo (Ed.), Envejecimiento activo $y$ actividades socioeducativas con personas mayores. (pp. 11-34). Madrid: Editorial Médica Panamericana

Consejo Nacional de Población (2012). Proyecciones de la población de México 20102050. México: CONAPO

Dirección de Educación (2019). PAS - Programa Aprender Siempre. Recuperado de https://www.mec.gub.uy/mecweb/mec201
$7 /$ container.jsp?contentid $=11451 \&$ site $=5 \& \mathrm{c}$ hannel $=$ mec\&breadid $=584 \& 3$ colid $=11451$

Facultad Latinoamericana de Ciencias Sociales (2008). Evaluación de consistencia y resultados del programa de atención a la demanda de educación para adultos a través del modelo de educación para la vida y el trabajo del INEA. Recuperado de http://www.sep.gob.mx/work/models/sep 1/Resource/102508/2.pdf

García, N. R. (2007). La educación con personas mayores en una sociedad que envejece. Horizontes Educacionales, 12(2), 51-62. Recuperado de http://www.redalyc.org/articulo.oa?id=979 17592006

Instituto de Estadística y Geografía (2019). Estadísticas a propósito del día internacional de las personas de edad ( $1^{\circ}$ de octubre). Recuperado de https://www.inegi.org.mx/contenidos/sala deprensa/aproposito/2019/edad2019_Nal. pdf

Instituto Nacional de las Personas Adultas Mayores (2010). Ejes rectores de la política pública nacional a favor de las personas adultas mayores. México: Instituto Nacional de las Personas Adultas Mayores

Medina, B., Llorent, V. J. y Llorent, V. (2013). Evolución y concepto de la educación permanente en España. Revista de Ciencias Sociales, 19(3), 511-522. Recuperado de http://www.redalyc.org/articulo.oa?id=280 28572013

Ministerio de Educación (2012). Programa de Alfabetización y Educación Básica para la población Joven y Adulta. Recuperado de https://www.mined.gob.sv/programas/pro gramas-educativos/item/5481-programade-alfabetizacion-y-educacion-basica-parala-poblacion-joven-y-adulta.html 
Ministerio de Educación (s.f.). ¿Qué es PRONEA? Recuperado de https://digeex.net/pronea/

Ministerio de Educación (s.f.). Currículo para Educación Extraordinaria para Personas con Escolaridad Inconclusa. https://educacion.gob.ec/curriculoeducacion-extraordinaria/

Ministerio de Educación Pública (2019). Instituto de Educación Comunitaria (IPEC). Recuperado de https://www.mep.go.cr/programas-yproyectos/yo-me-apunto/ipec

Ministerio de Educación y Ciencias (s.f.). Educación Permanente. Recuperado de https://www.mec.gov.py/cms/

Ministerio del Poder Popular para la Educación (2008). El desarrollo y el estado de la cuestión sobre el aprendizaje y la educación de adultos. Recuperado de https://uil.unesco.org/fileadmin/multime dia/uil/confintea/pdf/National_Reports/L atin\%20America\%20$\% 20$ Caribbean/Venezuela.pdf

Mogollón, E. (2012). Una perspectiva integral del adulto mayor en el contexto de la educación. Revista Interamericana de Educación de Adultos, 34(1), 56-74. Recuperado de https://www.redalyc.org/pdf/4575/4575 45090005.pdf

Organización Mundial de la Salud (2010). Día Internacional de las Personas de Edad - $1^{\circ}$ de octubre. Recuperado de https://www.who.int/features/qa/72/es/

Osorio, O. (2016). Envejecimiento poblacional: discriminación y políticas públicas integrales. Iztapalapa Revista de Ciencias Sociales y Humanidades, 81, 133-163. Recuperado de http://www.scielo.org.mx/pdf/izta/v37n8 1/2007-9176-izta-37-81-133.pdf
Razo-González, A. M. (2014). La política pública de vejez en México: de la asistencia pública al enfoque de derechos. CONAMED, 19(2), 78-85. Recuperado de https://www.medigraphic.com/cgi$\mathrm{bin} / \mathrm{new} /$ resumen.cgi?IDARTICULO=5756 9

Reyes, F. y Machado, E. (2017). Fundamentos teóricos- metodológicos sobre la educación del adulto mayor en el contexto de la EP. Humanidades Médicas, 17(2), 291-305. Recuperado de http://scielo.sld.cu/scielo.php?script=sci_a rttext\&pid=S1727-81202017000200004

Roque, Y. Azcuy, L. \& Toledo, L. (2015). Política social para la atención a la vejez: una necesidad para Villa Clara. Novedades en población, 6(22), 20-28. Recuperado de http://scielo.sld.cu/scielo.php?script=sci_a rttext\&pid=S1817-40782015000200003

Sabán, C. (2010). "Educación permanente" y "aprendizaje permanente": dos modelos teórico-aplicativos diferentes. Revista Iberoamericana de Educación, 52, 203-230. Recuperado de https://rieoei.org/RIE/article/view/617

Serdio, C. (2015). Educación y envejecimiento: una relación dinámica y en constante transformación. Educación XX1, 18(2), 237$255 . \quad$ Recuperado de https://www.redalyc.org/pdf/706/70638 708010.pdf

Tünnermann, C. (2010). La educación permanente y su impacto en la educación superior. Revista Iberoamericana de Educación Superior, 1(1), 120-133. Recuperado de http://www.scielo.org.mx/scielo.php?scrip $\mathrm{t}=$ sci_abstract\&pid=S2007$28722010000100008 \& \operatorname{lng}=e s \& n r m=i s o \&$ tl ng=en 
Valle, J. E. (2014). Educación permanente: los programas universitarios para mayores en España como respuesta a una nueva realidad social. Revista de la Educación Superior, 43(3), 117-138. Recuperado de https://www.sciencedirect.com/science/a rticle/pii/S0185276015000357
Valcárcel, M. J. (2003). Educación permanente y educación social: controversias y compromisos. Arxius de sociología, 8, 181$183 . \quad$ Recuperado de https://dialnet.unirioja.es/servlet/articulo ?codigo $=2237072$ 